Scientific Paper

\title{
Assessment of myocardial metabolic rate of glucose by means of Bayesian ICA and Markov Chain Monte Carlo methods in small animal PET imaging
}

\author{
Khadidja BERRADJA ${ }^{1, a}$, Nabil BoughanMI ${ }^{2}$ \\ ${ }^{1}$ University Abdelhamid Ibn Badis, Mostaganem, Algeria \\ ${ }^{2}$ University of Sciences and Technology, Oran, Algeria \\ ${ }^{a}$ E-mail address: berradja2@yahoo.fr
}

(received 21 April 2016; revised 12 June 2016; accepted 23 August 2016)

\begin{abstract}
In dynamic cardiac PET FDG studies the assessment of myocardial metabolic rate of glucose (MMRG) requires the knowledge of the blood input function (IF). IF can be obtained by manual or automatic blood sampling and cross calibrated with PET. These procedures are cumbersome, invasive and generate uncertainties. The IF is contaminated by spillover of radioactivity from the adjacent myocardium and this could cause important error in the estimated MMRG. In this study, we show that the IF can be extracted from the images in a rat heart study with 18F-fluorodeoxyglucose (18F-FDG) by means of Independent Component Analysis (ICA) based on Bayesian theory and Markov Chain Monte Carlo (MCMC) sampling method (BICA). Images of the heart from rats were acquired with the Sherbrooke small animal PET scanner. A region of interest (ROI) was drawn around the rat image and decomposed into blood and tissue using BICA. The Statistical study showed that there is a significant difference $(\mathrm{p}<0.05)$ between MMRG obtained with IF extracted by BICA with respect to IF extracted from measured images corrupted with spillover.
\end{abstract}

Key words: PET; input function; Bayesian ICA; MCMC; MMRG.

\section{Introduction}

Positron emission tomography (PET) imaging relies on the detection of positron annihilation photons issued by a radiotracer concentration in tissue. The labeled molecules with positron emitter isotopes allow to dynamically image the biochemistry in the body. Parameters of interest such as glucose metabolism can be calculated by means of pharmacokinetic models. They are crucial to assess the viability of a tissue, the follow up of patients, the study of drugs, a comparison between groups etc. In order to calculate these parameters, the quantity of the radiotracer supplied to the tissue needs to be known. It is the concentration of the radiotracer in the blood plasma or input function (IF). Pharmacokinetic modeling in PET imaging is based on the measurement of IF and the time-activity curve (TAC) of the target tissue. These two curves are related within a mathematical model that describes the biochemical behavior of the radioactive tracer in the tissues. IF can be assessed by manual or automatic blood sampling [1-3] which is risky, invasive, cumbersome and inaccurate. The samples need to be analyzed for radiotracer concentration in plasma and cross calibrated with the PET scanner.

For small animal 18F-FDG PET studies, the blood sampling procedure is more difficult and challenging because of the small size of blood vessels and the limited blood volume, the time delay and dispersion in the tubing, effect of the noise, cross-calibration between samples and the PET scanner $[2,4]$. The simplest technique is to extract IF from the images by means of region of interest (ROI) drawn on the blood pool in the case of cardiac imaging. In small animal imaging, hearts and arteries are small compared to the scanner spatial resolution. Hence, vascular radioactivity is blurred into adjacent tissues and vice versa. Therefore, curves obtained from regions drawn over the vascular space are a mixture of the IF and the contiguous tissues. Consequently IF is affected by spillover and partial volume effect (PVE) [5,6]. Meanwhile it is possible to mathematically decompose the measured images in images of blood and tissue. Factor analysis (FA) has been proposed to extract the input function and the tissue time activity curve from the dynamic PET imaging [7-10]. Although such factor analysis is considered an attractive tool to process dynamic image sequences, additional assumptions of a priori knowledge are needed to overcome the non-uniqueness of the solution [7]. In recent times, Independent Component Analysis (ICA) received great interest in biomedical signals. In PET imaging, some works [11-18] have been published reporting usage of ICA in image decomposition, image segmentation or for seeking of blood sampling from the images. Recently, Mabrouk et al [18] used ICA to extract the IF and the 
myocardium TACs directly from dynamic positron emission tomography (PET) images in small animals. The method assumes a super-Gaussian distribution model for the blood activity, and a sub-Gaussian distribution model for the tissue activity.

In this work we propose a method based on the Bayesian Independent Component Analysis and Markov Chain Monte Carlo methods (BICA) to extract IF from the images in a rat heart study with 18F-fluorodeoxyglucose (18F-FDG). A sequence of PET images is decomposed in a number of desired components with BICA to produce the input function in a simple and non invasive fashion. The principal advantage of this approach is its capability to incorporate non-negative constraints in the form of priors for both source signals and elements of the mixing matrix, and also it can produce a non square mixing matrix and these two characteristics makes this approach flexible and effective.

This approach was first used and has succeeded to solve the problem of non-negative source separation in a Bayesian framework for an application to the analysis of mixtures in spectroscopy [19]. We used it later in the last work [20]. We determine IF from internal artery in fluorodeoxyglucose (FDG) brain images by means of BICA and we have showed that BICA is a strong approach in FDG brain image decomposition to extract blood activity curves in a noninvasive way.

\section{Materials and Methods}

\section{Dynamic PET data model}

In dynamic PET images, each voxel contains two components: blood vessels and myocardium tissues. Hence, the radioactivity of a voxel is assumed to be a linear combination of the radioactivity in the two components [13]. The mixing process is then:

$X=A S+n$

Eq. 1

Where $X(N, F)$ is a dynamic PET data set consisting of $\mathrm{N}$ voxels with $F$ time scanned frames, each column of which is the scanned image at one time. $S(M, F)$ is an independent source image matrix which rows represent the blood volume image and the tissue volume image, so, in our case $\mathrm{M}=2$. The matrix $A(N, M)$ is combination coefficient matrix in which columns stand for the corresponding time-activity curves, for example the input blood curve and the output tissue curve, and $n(N, F)$ is the noise modeled as Gaussian with zero mean and covariance matrix $\Omega$. In the basic model of ICA, $X, S$ and $A$ represent respectively the observed signals, source signals composing $X$ and an unknown real mixing matrix.

\section{Bayesian approach}

The basic principle of the Bayesian approach to source separation is to take into account any information previously available on the source signals and mixing coefficients. The problem is formulated in a probabilistic framework by assigning prior distributions to the different variables. Bayes theorem allows describing the probability of the model in terms of the likelihood of the data and the prior probability of the model and the data. The posterior probability is then given by [19]:

$p(S, A \mid \mathrm{X})=\frac{p(X \mid S, A) P(S) P(A)}{p(X)}$

where the independence between $A$ and $S$ is assumed. As $p(\mathrm{X})$ is normalization constant, we can write:

$$
p(S, A \mid \mathrm{X}) \propto p(X \mid S, A) P(S) P(A)
$$

The first task of the inference is to encode our knowledge on the noise sequences, source signals and mixing coefficients by appropriate probability distributions.

\section{Noise distribution, priors on source signals and mixing coefficients}

The additive noise is assumed independent and identically distributed (i.i.d), independent of the source signals, stationary and Gaussian with zero mean and variances $\phi_{n}=$ $\left[\sigma_{1}^{2}, \sigma_{2}^{2}, \ldots, \sigma_{N}^{2}\right]$, therefore [19]:

$n \sim \prod_{i=1}^{N} \prod_{k=1}^{F} N_{G}\left(n_{i k} ; 0, \sigma_{i}^{2}\right)$

Eq. 4

where $N_{G}$ is a shorthand for a multivariate Gaussian distribution. The likelihood [19] is given by:

$$
p\left(X \mid A, S, \phi_{n}\right)=\prod_{i=1}^{N} \prod_{k=1}^{F} N_{G}\left(x_{i k} ; \sum_{j=1}^{M} a_{i j} s_{j k}, \sigma_{i}^{2}\right)
$$

To take into account the non-negativity, the sources are distributed as a Gamma distribution with parameters $\left(\alpha_{j}, \beta_{j}\right)$ which are supposed constant for each source. However, they may be different from one source to another. The sources are assumed mutually statistically independent. In the same manner, each column $\mathrm{j}$ of the mixing matrix is also assumed distributed as a Gamma distribution with parameters $\left(\gamma_{j}, \lambda_{j}\right)$. The advantage of the Gamma distribution is that its shape allows a better fit to the time-activity curves of blood and tissue. The prior densities on the source signals and the mixing matrix are:

$$
\begin{aligned}
& p\left(S \mid \phi_{s}\right)=\prod_{j=1}^{M} \prod_{k=1}^{F} G\left(s_{j k} ; \alpha_{j}, \beta_{j}\right) \\
& p\left(A \mid \phi_{a}\right)=\prod_{i=1}^{N} \prod_{j=1}^{M} G\left(a_{i j} ; \gamma_{j}, \lambda_{j}\right)
\end{aligned}
$$

Where

$\phi_{s}=\left[\alpha_{1}, \ldots, \alpha_{M}, \beta_{1}, \ldots, \beta_{M}\right]^{T}, \phi_{a}=\left[\gamma_{1}, \ldots, \gamma_{M}, \lambda_{1}, \ldots, \lambda_{M}\right]^{T}$ correspond to the parameters of the Gamma distributions and $\mathrm{G}$ stands for Gamma distribution. 
$\mathrm{G}$ is given by:

$G(x ; \alpha, \beta)=\left\{\begin{array}{lll}\frac{\beta^{\alpha}}{\Gamma(\alpha)} x^{\alpha-1} \exp (-\beta x) & \text { for } & x \geq 0, \\ 0 & \text { for } & x<0\end{array}\right.$

Eq. 8

Where $\Gamma(\alpha)$ is the Gamma function.

Replacing the set of hyperparameters by $\phi=\left\{\phi_{s}, \phi_{a}, \phi_{n}\right\}$, which are the unknown parameters of noise variances and Gamma densities, the Equation 3 becomes [19]:

$$
p(S, A \mid \mathrm{X}, \phi) \propto p\left(X \mid S, A, \phi_{n}\right) p\left(S \mid \phi_{s}\right) p\left(A \mid \phi_{a}\right)
$$

As the hyperparameters of the noise variances and the prior distributions are unknown (unsupervised learning), they have also to be inferred. Then the Equation 9 is expressed as:

$p(S, A, \phi \mid \mathrm{X}) \propto p\left(X \mid S, A, \phi_{n}\right) p\left(S \mid \phi_{s}\right) p\left(A \mid \phi_{a}\right) p(\phi) \quad$ Eq. 10

This joint posterior density is in general difficult and impossible in some cases to compute. In this paper, stochastic simulation tools is used to compute this joint posterior density by applying Monte Carlo Markov chain methods.

\section{Simulation and estimation via MCMC methods}

The objective of Monte Carlo Markov chain [21,22] is to simulate data distributed according to the joint posterior density, and then construct estimators based on these data. Sampling the posterior distribution in Equation 10 is achieved by using Gibbs algorithm and constructing the estimator from the samples of the Markov chain using the marginal posterior mean (MPM) estimator [19] as:

$\hat{S}=E[S \mid X], \hat{A}=E[A \mid X]$ et $\hat{\phi}=E[\phi \mid X]$

Eq. 11

The main steps of the algorithm to simulate $p(S, A, \phi \mid \mathrm{X})$, at each iteration $h$, are:

1. Simulating the source $S^{h+1}$ from

$p\left(S \mid \mathrm{X}, \mathrm{A}^{\mathrm{h}}, \phi^{\mathrm{h}}\right) \propto p\left(X \mid S, A^{h}, \phi^{h}\right) p\left(S \mid \phi^{h}\right)$

Eq. 12

2. Simulating the mixing coefficients $A^{h+1}$ from

$p\left(A \mid \mathrm{X}, \mathrm{S}^{\mathrm{h}+1}, \phi^{\mathrm{h}}\right) \propto p\left(X \mid S^{h+1}, A, \phi^{h}\right) p\left(A \mid \phi^{h}\right)$

Eq. 13

3. Simulating the hyperparameters of noise $\phi_{n}^{h+1}$ from

$p\left(\phi_{n} \mid \mathrm{X}, \mathrm{S}^{\mathrm{h}+1}, A^{\mathrm{h}+1}\right) \propto p\left(X \mid S^{h+1}, A^{h+1}, \phi_{n}\right) p\left(\phi_{n}\right)$

Eq. 14

4. Simulating the source hyperparameters $\phi_{s}^{h+1}$ from

$p\left(\phi_{s} \mid S^{h+1}\right) \propto p\left(S^{h+1} \mid \phi_{s}\right) p\left(\phi_{s}\right)$

Eq. 15

5. Simulating the mixing coefficients hyperparameters $\phi_{a}^{h+1}$ from

$p\left(\phi_{a} \mid A^{h+1}\right) \propto p\left(A^{h+1} \mid \phi_{a}\right) p\left(\phi_{a}\right)$

Eq. 16
The algorithm is iterated several times in order to have $h \max$ samples of $S^{h}, A^{h}$ and $\phi^{h}$. Estimates of the source signals, the mixing coefficients and the hyperparameters are obtained by MPM estimator [19] as:

$$
\begin{aligned}
& \hat{S}=\frac{1}{h \max -h \min } \sum_{h=h \min +1}^{h \max } S^{h} \quad \hat{A}=\frac{1}{h \max -h \min } \sum_{h=h \min +1}^{h \max } A^{h}, \\
& \hat{\phi}=\frac{1}{h \max -h \min } \sum_{h=h \min +1}^{h \max } \phi^{h}
\end{aligned}
$$

where $h$ min is the number of iterations corresponding to the burn-in run of the Markov chain whose related samples are discarded. The expressions of the conditional posterior distributions and their simulation techniques are detailed in [19].

\section{PET measurements}

The measurements were performed with the Sherbrooke small animal PET scanner [23]. Normal rats of about $300 \mathrm{~g}$ were measured for the heart after a nearly $5 \mathrm{mCi}$ bolus injection of 18F-FDG ( 7 rats). The temperature and the heart beats were continuously under control during the measurements. The rats were allowed free access to food and water before the measurements. 18F-FDG was measured during $30 \mathrm{~min}$ and this time slot was decomposed in 25 time frames of, respectively 12 x 5 seconds; 8 x 30 seconds and 5 x 300 seconds.

\section{PET kinetic modeling}

In order to calculate MMRG from the images, kinetic models were used where the time course of a tissue should fit a model. Figure 1 and Equations 18-20 summarize the procedure of TAC modeling in the case of FDG. The model in Figure 1 is translated in differential equations [24] as:

$$
\begin{aligned}
& \frac{d C_{f}(t)}{d t}=K_{1} C_{p}(t)-\left(k_{2}+k_{3}\right) C_{f}(t)+k_{4} C_{m}(t) \\
& \frac{d C_{m}(t)}{d t}=k_{3} C_{f}(t)-k_{4} C_{m}(t)
\end{aligned}
$$

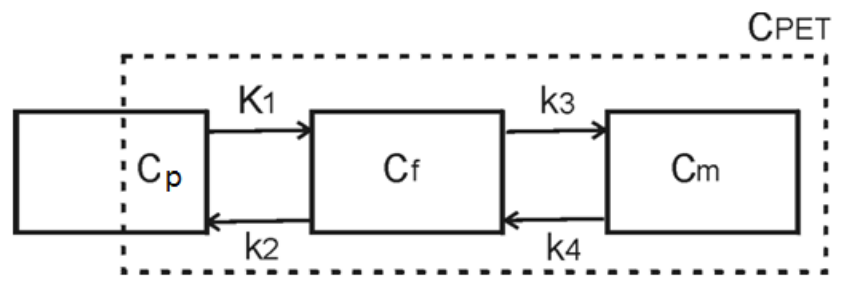

Figure 1. Diagram of the three-compartment model for 18F-FDG illustrating radiotracer in blood $\left(C_{p}\right)$, free $18 F$ FDG $\left(C_{f}\right)$ and metabolized 18F-FDG $\left(C_{m}\right)$ compartments, the perfusion constant $\left(K_{1}\right)$ and the rate constants $\left(k_{2}-k_{4}\right)$. The dashed line corresponds to the tissue ROI measured with PET ( $\mathrm{C}_{\text {PET }}$ or tissue TAC). 
The solutions to the differential equations are given by:

$C_{f}(t)=\frac{K_{1}}{\alpha_{2}-\alpha_{1}}\left[\left(k_{4}-\alpha_{1}\right) e^{-\alpha_{1} t}+\left(\alpha_{2}-k_{4}\right) e^{-\alpha_{2} t}\right] \otimes C_{p}(t)$

$C_{m}(t)=\frac{K_{1} k_{3}}{\alpha_{2}-\alpha_{1}}\left(e^{-\alpha_{1} t}-e^{-\alpha_{2} t}\right] \otimes C_{p}(t)$

The PET measurement is described by:

$$
\begin{aligned}
C_{P E T}(t)= & \frac{K_{1}}{\alpha_{2}-\alpha_{1}}\left[\left(k_{3}+k_{4}-\alpha_{1}\right) * \exp \left(-\alpha_{1} * t\right)+\right. \\
& \left.\left(\alpha_{2}-k_{3}-k_{4}\right) * \exp \left(-\alpha_{2} * t\right)\right] \otimes C_{p}(t)+k_{5} * C_{p}(t)
\end{aligned}
$$

Where $k_{5}$ is a fraction $\left(0 \leq k_{5}<1\right)$ of $C_{p}$ called blood volume and $\alpha_{1}, \alpha_{2}$ are combinations of the time constants as follows:

$\alpha_{1}=\left[k_{2}+k_{3}+k_{4}-\sqrt{\left(k_{2}+k_{3}+k_{4}\right)^{2}-4 k_{2} k_{4}}\right] / 2$

$\alpha_{2}=\left[k_{2}+k_{3}+k_{4}+\sqrt{\left(k_{2}+k_{3}+k_{4}\right)^{2}-4 k_{2} k_{4}}\right] / 2 \quad$ Eq. 21

The injected radiotracer is found first in blood (compartment $C_{p}$ in Figure 1), then it crosses the capillaries towards the cells $\left(C_{f}\right)$ before being metabolized $\left(C_{m}\right)$. This transfer is governed by rate constants $K_{1}-k_{4}$. The compartment model is then translated into equations where $C_{p}$ represents IF. $C_{P E T}$ in Equation 20 should fit the measured tissue TAC. Knowing $C_{p}$ and $C_{P E T}$ on several time point, the rate constants $K_{1}-k_{4}$ are obtained by nonlinear least squares fitting. The rate constants allow to calculate MMRG as:

$M M R G=\frac{g l}{L C} \frac{K_{1} k_{3}}{k_{2}+k_{3}}$

where $g l$ is the concentration of glucose in plasma and $L C$ is the lumped constant taken as 1 .

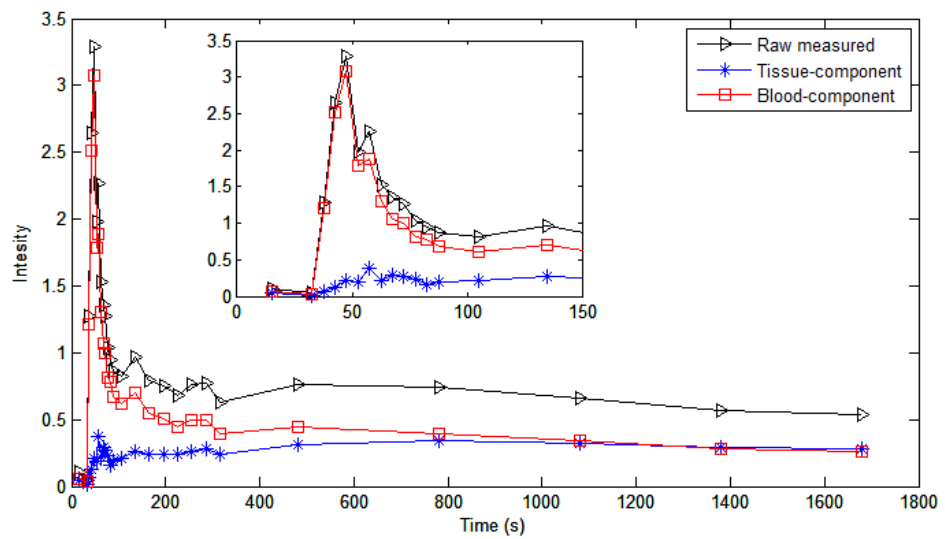

\section{Results}

In order to define the time course of the radiotracer concentration in blood and tissue, three regions of interest (ROI) were drawn. The first one included the whole rat image to be decomposed into two images of tissue and blood. The second ROI was drawn around the blood chamber to account for blood time-activity curve (TAC). The third ROI was drawn on myocardium to delimit tissue TAC.

Figure 2 shows the rat heart measured with 18F-FDG and decomposed with BICA in tissue and blood images. Figure 2 top-left is the measured image at the last time frame. Figure 2 top-right is the same image as top-left with the ROIs indicating the pixels averaged to produce tissue and blood TACs. Figure 2 bottom-left is the image extracted with BICA representing blood distribution. Figure 2 bottom-right displays the tissue component separated from blood component. One can notice on tissue image the reduced radioactivity at the ventricle chamber which is the site of blood.

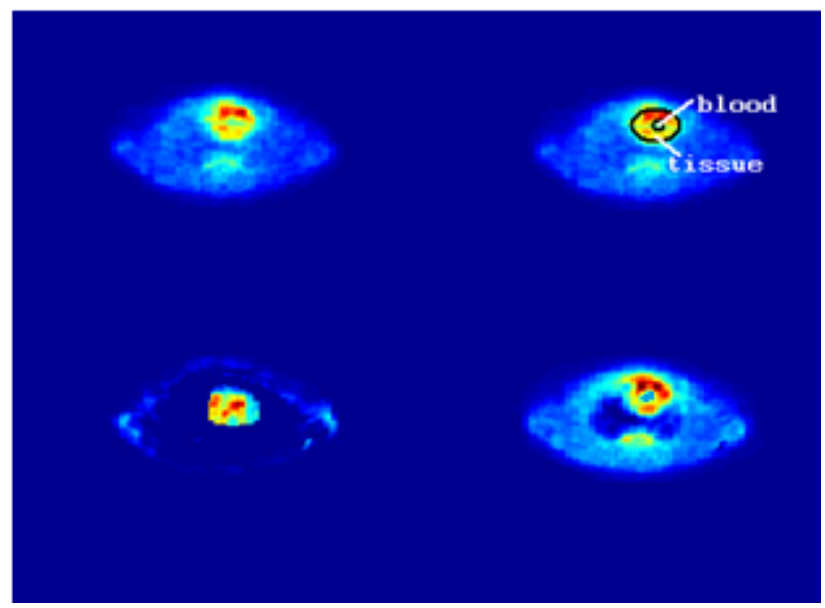

Figure 2. Decomposition of 18F-FDG images. Top row: measured image and same image with tissue and blood ROIs. Bottom: blood (left) and tissue (right) images obtained with BICA.

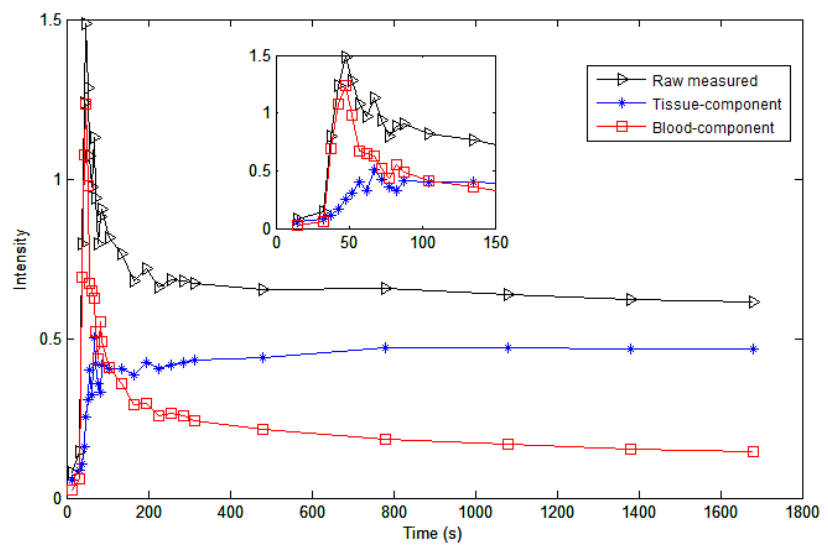

Figure 3. 18F-FDG time-activity curves of blood (left) and tissue (right) components as calculated with BICA in comparison to ROI on measured image (Raw measured). 
By applying the ROIs on the three sets of dynamic images (raw measured, blood and tissue components), we obtain the blood and tissue TACs as shown in Figure 3. In these figures, the blood and tissue TACs are respectively compared to the TACs obtained from the measured images. We observe that the method was able to decompose the images as there is nearly no blood contribution in tissue TAC and reciprocally, there is nearly no tissue contribution in blood TAC. This observation can be supported by the fact that the radiotracer injection was a bolus and at early time of the TAC, the measured signal was almost from the blood component. Furthermore, at later times, the radioactivity in blood is low depending on recirculation only while the major contribution results from tissue. The ratio of integrals of calculated blood TAC with respect to the measured blood TAC (Measured-Calculated)/Measured) was found $69.63 \%$. In this case, the recovery of intensity was minor as the heart was enough large to be subject to PVE. That would not be the case for the heart of a mouse which is approximately ten times smaller where the pixels are formed by a mixture of tissue and blood signals.

Knowing the blood TAC without contamination from tissue, it is used as IF in Equation 20 together with the measured tissue TAC to produce the rate constants. The fit of the model to the data is shown in Figure 4. The values of MMRG obtained with both methods are reported in Figure 5. We have used Wilcoxon signed-rank test to compare MMRG obtained with both methods. The results obtained show a significant difference $(\mathrm{p}=0.0156)$ between MMRG obtained with BICA and ROI. Thus, there is a significant gain of MMRG with IF extracted by BICA with respect to IF extracted from measured images.

\section{Discussion}

Blood sampling is mandatory in PET kinetic modeling in order to calculate the physiological parameters. IF can be determined from blood samples which are invasive, hard to obtain especially from patients, children and small animals, hard to analyse where small amounts of nearly 25 samples have to measured with time, centrifugated, plasma extracted, measured for radioactivity and cross calibrated with the PET scanner. The automatic blood sampling presents the same difficulties except the risk of the operator error, and both of them introduce uncertainties. The most common method used is to extract IF from images by drawing ROIs around blood vessels. However, IF is affected by spillover and partial volume effect. To resolve the spillover problem, BICA is applied to extract IF from a dynamic sequence of PET images. This method first requires the selection of a priori suitable for the unknown parameters. For the distribution of sources and mixing coefficients we considered a model as gamma prior density. The choice of the gamma density is justified firstly by the consideration of the constraint of non-negativity for the sources and the mixing coefficients and on the other by the similarity of this law to time-activity curves (TAC) of blood and tissue. Both source signals and mixing coefficients were estimated successfully without negative values. BICA has already shown its efficacy in brain imaging and in the present study, we demonstrated that Bayesian ICA and MCMC sampling succeeded once again in decomposing 18F-FDG cardiac images in small animal where the resolution is very small $(\sim 1 \mathrm{~mm})$ and the PVE is very important. BICA can be used at least for imaging with radiotracers like $18 \mathrm{~F}-\mathrm{FDG}$ which provides contrasted images as a function of measurement time, and producing non correlated blood and tissue signals. Since BICA provides blood images by decomposing 18F-FDG images, this approach could be used to generate blood volume images to locate vasculature deficiencies or pathologies.

The tissue and blood components are strong components (several voxels with correlated time-course intensities) and are thus clearly identified. However, other components possibly constituting secondary components and noise can be associated with the two main components at separate positions. Thus, the appearance of some voxels at the contour of the body in lowerleft image of Figure 2.

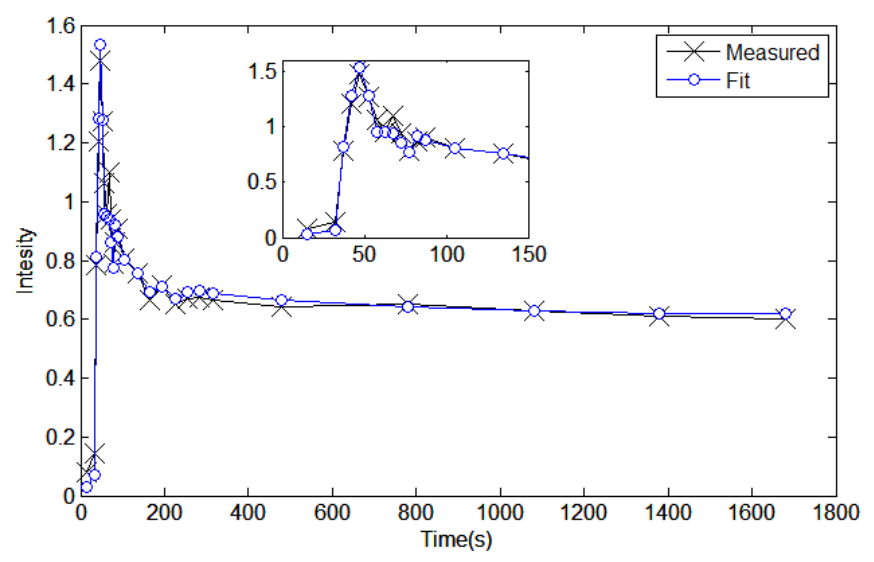

Figure 4. Fit of the model to the tissue TAC.

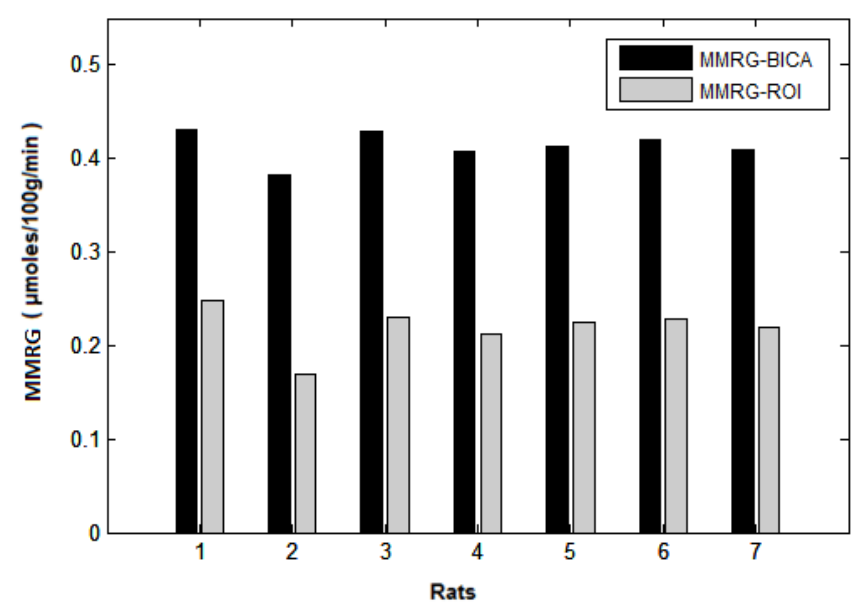

Figure 5. The MMRG values obtained with both methods BICA and ROI. 
The extracted IF from blood images was free from time delay, dispersion and tissue to blood spillover, makes PET imaging less invasive, more precise in the sense that IF and the TACs are both in the same units with no extra measurements, easy and safe in the experimental procedures for the personnel and the patients. The proposed algorithm is flexible, performing and does not require preprocessing and spatial whitening of observations, the only necessary parameters to provide are the number of independent components. The suppression of noise is taken into account in the process of separation.

\section{Conclusions}

We applied in this paper a method based on the joint use of Bayesian ICA and MCMC sampling to extract IF from small animals PET imaging. The results obtained illustrate once again the strength and the effectiveness of BICA to decompose a sequence of PET images in a number of desired components and to produce the input function in a simple and non invasive fashion. Finally, the validity of the approach on other radiotracers remains to be confirmed.

\section{References}

[1] Senda M, Nishizawa S, Yonekura Y, et al. Measurement of arterial time-activity curve by monitoring continuously drawn arterial blood with an external detector: errors and corrections. Ann Nucl Med. 1988;2(1):7-12.

[2] Boellaard R, Van Lingen A, Van Balen SC, et al. Characteristics of a new fully programmable blood sampling device for monitoring blood radioactivity during PET. Eur J Nucl Med. 2001; 28(1):81-89.

[3] Convert L, Morin-Brassard G, Cadorette J, et al. A new tool for molecular imaging: the microvolumetric Beta blood counter. J Nucl Med. 2007;48(7):1197-1206.

[4] Ranicar AS, Williams CW, Schnorr L, et al. The on-line monitoring of continuously withdrawn arterial blood during PET studies using a single BGO/photomultiplier assembly and non-stick tubing. Med Prog Technol. 1991;17(3-4):259-264.

[5] Lin KP, Huang SC, Choi Y, Brunken, et al. Correction of spillover radioactivities for estimation of the blood time-activity curve from the imaged LV chamber in cardiac dynamic FDG PET studies. Phys Med Biol. 1995;40(4):629-642.

[6] Mourik JE, Lubberink M, Schuitemaker A, et al. Image-derived input functions for PET brain studies. Eur J Nucl Med Mol Imaging. 2009;36(3): 463-471.

[7] Houston AS. The effect of apex-finding errors on factor images obtained from factor analysis and oblique transformation. Phys Med Biol. 1984;29(9):1109-1116.

[8] Wu HM, Hoh CK, Choi Y, et al. Factor analysis for extraction of blood time-activity curves in dynamic FDG-PET studies. J Nucl Med. 1995;36(9):1714-1722.

[9] Bentourkia M. Kinetic modeling of PET-FDG in the brain without blood sampling. Comput Med Imaging Graph. 2006;30(8):447451.

[10] Buvat I, Benali H, Frouin F, et al. Target apex-seeking in factor analysis of medical image sequences. Phys Med Biol. 1993;38(1):123-138.

[11] Lee JS, Lee DS, Ahn JY, et al. Blind separation of cardiac components and extraction of input function from $\mathrm{H}_{2}{ }^{15} \mathrm{O}$ dynamic myocardial PET using independent component analysis. J Nucl Med. 2001;42(6):938-943.

[12] Naganawa M, Kimura Y, Ishii K, et al. Extraction of a plasma time-activity curve from dynamic brain PET images based on independent component analysis. IEEE Trans Biomed Eng. 2005;52(2):201-209.

[13] Naganawa M, Kimura Y, Ishii K, et al. Temporal and spatial blood information estimation using Bayesian ICA in dynamic cerebral positron emission tomography. Dig Sig Process. 2007; 17(5):979-993.

[14] Chen K, Chen X, Renaut R, et al. Characterization of the image-derived carotid artery input function using independent component analysis for the quantitation of [18F] fluorodeoxyglucose positron emission tomography images. Phys Med Biol. 2007;52(23):70557071.

[15] Su KH, Lee JS, Yang YW, et al. Partial volume correction of the microPET blood input function using ensemble learning independent component analysis. Phys Med Biol. 2009;54(6):1823-1846.

[16] Margadan-Mendez M, Juslin A, Nesterov SV, et al. ICA Based Automatic Segmentation of Dynamic $\mathrm{H}_{2}{ }^{15} \mathrm{O}$ Cardiac PET Images. IEEE Trans Info Tech Biomed. 2010;14(3):795-802.

[17] Fu Z, Tantawy MN, Peterson TE. Ensemble learning (EL) independent component analysis (ICA) approach to derive blood input function from FDG-PET images in small animal. IEEE Nucl Sci Symp Conf Rec. 2006;5:2708-2712.

[18] Mabrouk R, Dubeau F, Bentabet L. Dynamic cardiac PET imaging: extraction of time-activity curves using ICA and a generalized Gaussian distribution model. IEEE Trans Biomed Eng. 2012;60(1):63-71.

[19] Moussaoui S, Brie D, Mohammad-Djafari A, et al. Separation of non-negative mixture of non-negative sources using a Bayesian approach and MCMC sampling. IEEE Trans Sig Process. 2006;54(11):4133-4145. 
[20] Berradja K, Boughanmi N. Assessment of brain glucose metabolism with input function determined from Brain PET images by means of Bayesian ICA and MCMC methods. Comput Med Imaging Graph. 2012;36(8):620-626.

[21] Robert C. Monte Carlo Statistical Methods. Berlin: Springer-Verlag; 1999.

[22] Fitzgerald W. Markov chain Monte Carlo methods with applications to signal processing. Sig Process. 2001;81(1):3-18.

[23] Bergeron M, Cadorette J, Beaudoin J-F, et al. Evaluation of the Performance-Based Digital LabPET APD PET Scanner. IEEE Trans Nucl Sci. 2009;56(1).

[24] Phelps ME, Huang SC, Hoffman EJ, et al. Tomographic measurement of local cerebral glucose metabolic rate in humans with (F18)2-fluoro-2-deoxy-D-glucose: validation of method. Ann Neurol. 1979;6(5):371-388. 